\title{
AN ALGORITHM FOR A MULTIOBJECTIVE, MULTILEVEL LINEAR PROGRAMMING
}

\author{
Zhi-Wei Wang \\ Shinwa Kogyo Co. \\ Hiroyuki Nagasawa
Osaka Prefecture University \\ Noriyuki Nishiyama \\ Osaka Institute of Technology
}

(Received May 30, 1994; Final January 26, 1996)

\begin{abstract}
This paper discusses a solution method to a multiobjective, multi-level decentralized system. A decomposition method is proposed for dividing the $n$-level problem finally into $n-2$ single level problems and a two-level problem. A method with domination trees based on the "two-level simplex algorithm" is developed for generating the nondominated solutions to this type of $n$-level decentralized system.
\end{abstract}

\section{Introduction}

In a multi-level decentralized system consisting of one headquarters, several divisions and factories [2], it is required to make its decision considering not only a competition with others but also the coexistence with the social environment to pursuit the overall development of the corporation.

From the strategic view points, the headquarters is forced in such a circumstance to consider the second and third objectives in making its decision, while the objectives of divisions and factories remain single. This type of decentralized system is called a "multiobjective, multilevel decentralized system" in this paper.

A multi-level decentralized linear system can generally be represented as a multi-level linear programming problem [1] , but so far there is no solution method proposed for dealing with a multiobjective, multilevel decentralized system.

This paper first formulates a mathematical model for a multiobjective, multilevel decentralized system. A method for generating all the nondominated solutions to this model is proposed and a numerical example of a two-objective, there-level decentralized system is demonstrated to show the effectiveness of the proposed method.

\section{Model Formulation}

A corporation adopting a decentralized decision-making system can be described as a multi-level decentralized system as shown in Fig. 1. The first(top) level in Fig. 1 means the headquarters of the corporation and the second level, various divisions. The headquarters allocates gross resources such as budget, man power, energy, raw materials and facilities among the divisions. Each division makes an aggregate production plan and resource allocation for factories belonging to the division. Factories located at the third level shown in Fig. 1 produce different types of products, using the resources allocated by their divisions.

In general, a multiobjective, $n$-level decentralized system described above is mathematically formulated in the following aggregated form:

$\left[\right.$ Problem $\left.\mathbf{P}^{(n)}\right]$

$<$ the 1st level problem $>$ 


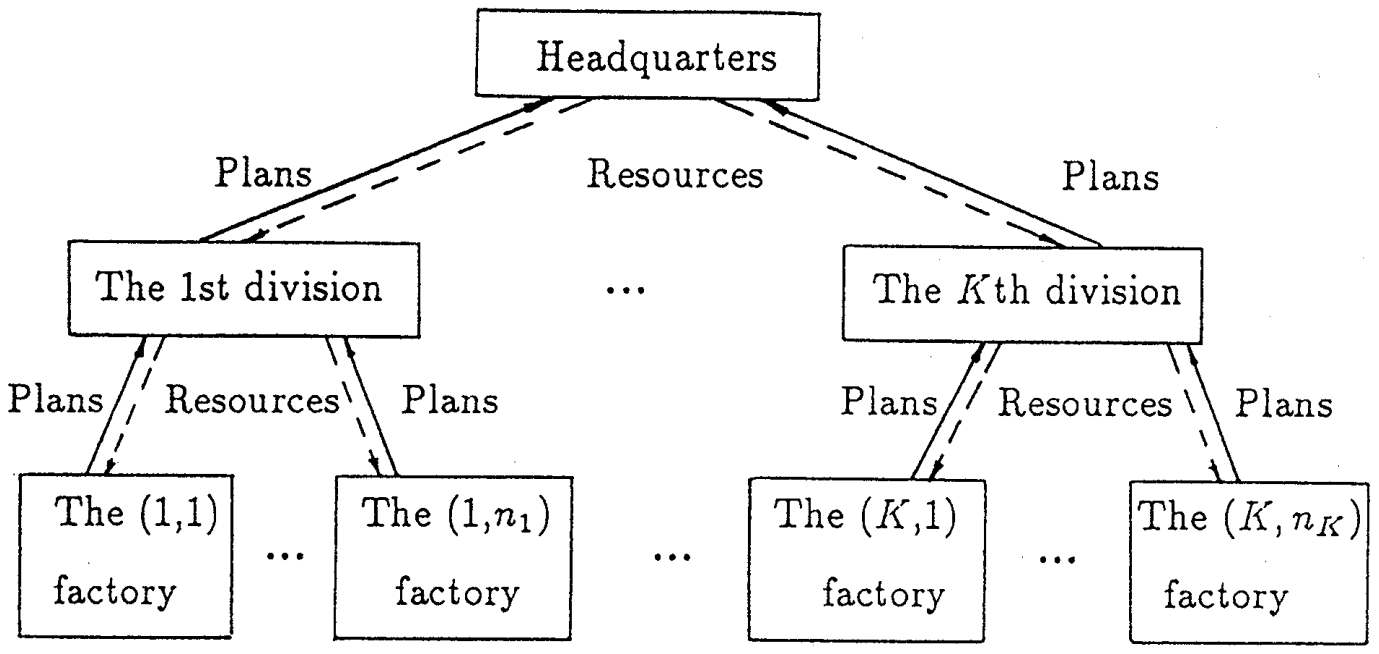

Fig.1 Organization of a three-level decentralized system

(-.- Flow of resource allocation plan. Flow of production plan)

Maximize $\quad f_{1}^{1}\left(\mathbf{y}_{1}\right)=\left\{\mathbf{c}_{1}^{1} \mathbf{x}\left(\mathbf{y}_{n-1}\left(\ldots\left(\mathbf{y}_{2}\left(\mathbf{y}_{1}\right)\right)\right)\right)-\mathbf{c}_{n-1,1}^{1} \mathbf{y}_{n-1}\left(\mathbf{y}_{n-2}\left(\ldots\left(\mathbf{y}_{2}\left(\mathbf{y}_{1}\right)\right)\right)\right)\right.$

$$
\left.-\mathbf{c}_{n-2,1}^{1} \mathbf{y}_{n-2}\left(\mathbf{y}_{n-3}\left(\ldots\left(\mathbf{y}_{2}\left(\mathbf{y}_{1}\right)\right)\right)\right)-\ldots-\mathbf{c}_{2,1}^{1} \mathbf{y}_{2}\left(\mathbf{y}_{1}\right)\right\}-\mathbf{c}_{1,1}^{1} \mathbf{y}_{1} \text {, }
$$

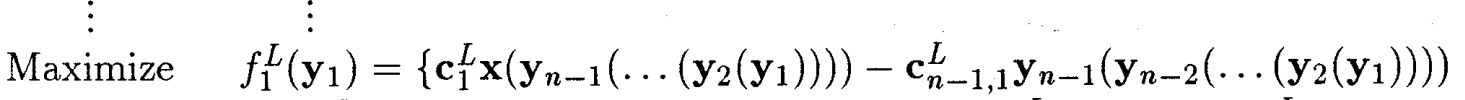

$$
\left.-\mathbf{c}_{n-2,1}^{L} \mathbf{y}_{n-2}\left(\mathbf{y}_{n-3}\left(\ldots\left(\mathbf{y}_{2}\left(\mathbf{y}_{1}\right)\right)\right)\right)-\ldots-\mathbf{c}_{2,1}^{L} \mathbf{y}_{2}\left(\mathbf{y}_{1}\right)\right\}-\mathbf{c}_{1,1}^{L} \mathbf{y}_{1} \text {, }
$$

subject to $\mathbf{G}_{1} \mathbf{y}_{1} \leqq \mathbf{Y}_{1}$,

$$
\mathbf{y}_{1} \geqq \mathbf{0}
$$

$<$ the 2nd level problem $>$

Maximize $\quad f_{2}\left(\mathbf{y}_{2}\right)=\left\{\mathbf{c}_{2} \mathbf{x}\left(\mathbf{y}_{n-1}\left(\ldots\left(\mathbf{y}_{3}\left(\mathbf{y}_{2}\right)\right)\right)\right)-\mathbf{c}_{n-1,2} \mathbf{y}_{n-1}\left(\mathbf{y}_{n-2}\left(\ldots\left(\mathbf{y}_{3}\left(\mathbf{y}_{2}\right)\right)\right)\right)\right.$

$$
\left.-\mathbf{c}_{n-2,2} \mathbf{y}_{n-2}\left(\mathbf{y}_{n-3}\left(\ldots\left(\mathbf{y}_{3}\left(\mathbf{y}_{2}\right)\right)\right)\right)-\ldots-\mathbf{c}_{3,2} \mathbf{y}_{3}\left(\mathbf{y}_{2}\right)\right\}-\mathbf{c}_{2,2} \mathbf{y}_{2} \text {, }
$$

subject to $\quad \mathbf{G}_{2} \mathbf{y}_{2} \leqq \mathbf{Y}_{2}+\mathbf{D}_{2} \mathbf{y}_{1}$,

$$
\mathrm{y}_{2} \geqq 0
$$

$<$ the $(n-1)$ th level problem $>$

Maximize $\quad f_{n-1}\left(\mathbf{y}_{n-1}\right)=\mathbf{c}_{n-1} \mathbf{x}\left(\mathbf{y}_{n-1}\right)-\mathbf{c}_{n-1, n-1} \mathbf{y}_{n-1}$

subject to

$$
\begin{aligned}
& \mathbf{G}_{n-1} \mathbf{y}_{n-1} \leqq \mathbf{Y}_{n-1}+\mathbf{D}_{n-1} \mathbf{y}_{n-2}, \\
& \quad \mathbf{y}_{n-1} \geqq \mathbf{0},
\end{aligned}
$$

$<$ the nth level problem $>$

Maximize $f_{n}(\mathbf{x})=\mathbf{c}_{n} \mathbf{x}$

subject to

$$
\begin{aligned}
\mathbf{A x} & =\mathbf{b}+\mathrm{D}_{n} \mathbf{y}_{n-1} \\
\mathbf{x} & \geqq 0
\end{aligned}
$$

where the notations are defined below.

$L$ : number of objective functions of the headquarters

$\mathbf{x}$ : production amount vector to be planned at the $n$th level (including slack variables if these are introduced to Eq. (12)) 
$\mathbf{x}\left(\mathbf{y}_{n-1}\right)$ : optimal solution to the $n$th level problem with the resource given by $\mathbf{y}_{n-1}$

$\mathbf{y}_{i}\left(\mathbf{y}_{i-1}\right)$ : optimal solution to the $i$-level problem with the resource given by $\mathbf{y}_{i-1}, i=$ $2,3, \ldots, n-1$

$\mathbf{x}\left(\mathbf{y}_{n-1}\left(\cdots\left(\mathbf{y}_{i+1}\left(\mathbf{y}_{j}\right)\right)\right)\right)$ : information on the optimal production amount determined at the $n$th level, fed back to the $j$ th level

$\mathbf{y}_{i}\left(\mathbf{y}_{i-1}\left(\cdots\left(\mathbf{y}_{j+1}\left(\mathbf{y}_{j}\right)\right)\right)\right)$ : information on the optimal resource allocation determined at the $i$ th level, fed back to the $j$ th level, $i=j+1, \ldots, n-1, j=1,2, \ldots, n-2$

$\mathbf{D}_{i}$ : constraint matrix with respect to the resource $\mathbf{y}_{i-1}$ allocated from the $(i-1)$ th level to the $i$ th level

A: technical coefficient matrix with respect to production amount vector $\mathbf{x}$ in the $n$th level problem

$\mathbf{G}_{i}$ : technical coefficient matrix with respect to resource allocation vector $\mathbf{y}_{i}$ in the $i$ th level problem

b: lower limit vector for resources available in the $n$th level problem

$\mathbf{Y}_{i}$ : lower limit vector for resources available in the $i$ th level problem

$\mathbf{c}_{1}^{l}$ : the $l$ th objective coefficient row vector with respect to $\mathbf{x}$ in the 1 st level problem

$\mathbf{c}_{i}$ : objective coefficient row vector with respect to $\mathbf{x}$ in the $i$ th level problem, $i=2,3, \ldots, n$

$\mathbf{c}_{i, j}$ : objective coefficient row vector with respect to $\mathbf{y}_{j}$ in the $i$ th level problem, $1 \leqq j \leqq i \leqq n-1$

If the decision space defined by Eqs. (3), (4), (6), (7), (9), (10), (12) and (13) is feasible, problem $\mathbf{P}^{(n)}$ has a set of nondominated solutions expressed by $\left(\mathbf{y}_{1}^{\dagger}, \mathbf{y}_{2}^{\dagger}, \ldots, \mathbf{y}_{n-1}^{\dagger}, \mathbf{x}^{\dagger}\right)$ satisfying the following three conditions:

(a) Eqs. (3), (4), (6), (7), (9), (10), (12) and (13) hold.

(b) $\left(\mathbf{y}_{i}^{\dagger}, \mathbf{y}_{i+1}^{\dagger}, \ldots, \mathbf{y}_{n-1}^{\dagger}, \mathbf{x}^{\dagger}\right)$ is optimal for the $i$ th level problem with resources given by

$\mathbf{y}_{1}^{\dagger}, \ldots, \mathbf{y}_{i-1}^{\dagger}$, that is, $\left(\mathbf{y}_{i}^{\dagger}, \mathbf{y}_{i+1}^{\dagger}, \ldots, \mathbf{y}_{n-1}^{\dagger}, \mathbf{x}^{\dagger}\right)=\left(\mathbf{y}_{i}\left(\mathbf{y}_{i-1}^{\dagger}\left(\ldots\left(\mathbf{y}_{2}^{\dagger}\left(\mathbf{y}_{1}^{\dagger}\right)\right)\right)\right)\right.$,

$\left.\mathbf{y}_{i+1}\left(\mathbf{y}_{i}^{\dagger}\left(\ldots\left(\mathbf{y}_{2}^{\dagger}\left(\mathbf{y}_{1}^{\dagger}\right)\right)\right)\right), \ldots, \mathbf{y}_{n-1}\left(\mathbf{y}_{n-2}^{\dagger}\left(\ldots\left(\mathbf{y}_{2}^{\dagger}\left(\mathbf{y}_{1}^{\dagger}\right)\right), \mathbf{x}\left(\mathbf{y}_{n-1} \ldots \mathbf{y}_{2}^{\dagger}\left(\mathbf{y}_{1}^{\dagger}\right)\right)\right)\right)\right)$,

$i=2,3, \ldots, n-1$.

(c) There is no feasible solution, $\left(\mathbf{y}_{1}, \mathbf{y}_{2}\left(\mathbf{y}_{1}\right), \ldots, \mathbf{y}_{n-1}\left(\mathbf{y}_{n-2}\left(\ldots\left(\mathbf{y}_{2}\left(\mathbf{y}_{1}\right)\right)\right)\right)\right.$,

$\left.\mathbf{x}\left(\mathbf{y}_{n-1}\left(\ldots\left(\mathbf{y}_{2}\left(\mathbf{y}_{1}\right)\right)\right)\right)\right)$, satisfying

$\mathbf{c}_{1}^{l} \mathbf{x}\left(\mathbf{y}_{n-1}\left(\ldots\left(\mathbf{y}_{2}\left(\mathbf{y}_{1}\right)\right)\right)\right)-\mathbf{c}_{n-1,1}^{l} \mathbf{y}_{n-1}\left(\mathbf{y}_{n-2}\left(\ldots\left(\mathbf{y}_{2}\left(\mathbf{y}_{1}\right)\right)\right)\right)-\ldots-\mathbf{c}_{2,1}^{l} \mathbf{y}_{2}\left(\mathbf{y}_{1}\right)-\mathbf{c}_{1,1}^{l} \mathbf{y}_{1}$

$\geqq \mathbf{c}_{1}^{l} \mathbf{x}^{\dagger}-\mathbf{c}_{n-1,1}^{l} \mathbf{y}_{n-1}^{\dagger}-\ldots-\mathbf{c}_{2,1}^{l} \mathbf{y}_{2}^{\dagger}-\mathbf{c}_{1,1}^{l} \mathbf{y}_{1}^{\dagger}, \quad l=1,2, \ldots, L$,

with at least one inequality becoming " $>$ ".

\section{Properties of the Model}

The multiobjective, multi-level decentralized system formulated in the previous section can be transformed into neither a usual multi-level decentralized system nor a centralized system with multiple objectives. To analyze the properties of problem $\mathbf{P}^{(n)}$ defined by Eqs. (1) through (13), we first decompose the problem $\mathbf{P}^{(n)}$ into the two problems $\mathbf{P}^{(n-1)}$ and $\mathbf{L} \mathbf{P}^{(n-1)}$ as follows:

$\left[\right.$ Problem $\left.\mathbf{P}^{(n-1)}\right]$

$<$ the reduced 1 st level problem $>$

Maximize $\quad f_{1}^{1}\left(\mathbf{y}_{1}, \mathbf{y}_{2}\right)=\left\{\mathbf{c}_{1}^{1} \mathbf{x}\left(\mathbf{y}_{n-1}\left(\ldots\left(\mathbf{y}_{3}\left(\mathbf{y}_{2}\right)\right)\right)\right)-\mathbf{c}_{n-1,1}^{1} \mathbf{y}_{n-1}\left(\mathbf{y}_{n-2}\left(\ldots\left(\mathbf{y}_{3}\left(\mathbf{y}_{2}\right)\right)\right)\right)\right.$

$\left.-\mathbf{c}_{n-2,1}^{1} \mathbf{y}_{n-2}\left(\mathbf{y}_{n-3}\left(\ldots\left(\mathbf{y}_{3}\left(\mathbf{y}_{2}\right)\right)\right)\right)-\ldots-\mathbf{c}_{3,1}^{1} \mathbf{y}_{3}\left(\mathbf{y}_{2}\right)\right\}$

$-\mathbf{c}_{2,1}^{1} \mathbf{y}_{2}-\mathbf{c}_{1,1}^{1} \mathbf{y}_{1}$, 
Maximize $\quad f_{1}^{L}\left(\mathbf{y}_{1}, \mathbf{y}_{2}\right)=\left\{\mathbf{c}_{1}^{L} \mathbf{x}\left(\mathbf{y}_{n-1}\left(\ldots\left(\mathbf{y}_{3}\left(\mathbf{y}_{2}\right)\right)\right)\right)-\mathbf{c}_{n-1,1}^{L} \mathbf{y}_{n-1}\left(\mathbf{y}_{n-2}\left(\ldots\left(\mathbf{y}_{3}\left(\mathbf{y}_{2}\right)\right)\right)\right)\right.$ $\left.-\mathbf{c}_{n-2,1}^{L} \mathbf{y}_{n-2}\left(\mathbf{y}_{n-3}\left(\ldots\left(\mathbf{y}_{3}\left(\mathbf{y}_{2}\right)\right)\right)\right)-\ldots-\mathbf{c}_{3,1}^{L} \mathbf{y}_{3}\left(\mathbf{y}_{2}\right)\right\}$ $-\mathbf{c}_{2,1}^{L} \mathbf{y}_{2}-\mathbf{c}_{1,1}^{L} \mathbf{y}_{1}$,

subject to

$$
\begin{aligned}
& \mathbf{G}_{1} \mathbf{y}_{1} \leqq \mathbf{Y}_{1}, \\
& \mathbf{G}_{2} \mathbf{y}_{2} \leqq \mathbf{Y}_{2}+\mathbf{D}_{2} \mathbf{y}_{1}, \\
& \mathbf{y}_{1}, \mathbf{y}_{2} \geqq \mathbf{0},
\end{aligned}
$$

$<$ the reduced 2nd level problem $>$

Maximize $\quad f_{3}\left(\mathbf{y}_{3}\right)=\left\{\mathbf{c}_{3} \mathbf{x}\left(\mathbf{y}_{n-1}\left(\ldots\left(\mathbf{y}_{4}\left(\mathbf{y}_{3}\right)\right)\right)\right)-\mathbf{c}_{n-1,3} \mathbf{y}_{n-1}\left(\mathbf{y}_{n-2}\left(\ldots\left(\mathbf{y}_{4}\left(\mathbf{y}_{3}\right)\right)\right)\right)\right.$
$\left.-\mathbf{c}_{n-2,3} \mathbf{y}_{n-2}\left(\mathbf{y}_{n-3}\left(\ldots\left(\mathbf{y}_{4}\left(\mathbf{y}_{3}\right)\right)\right)\right)-\ldots-\mathbf{c}_{4,3} \mathbf{y}_{4}\left(\mathbf{y}_{3}\right)\right\}-\mathbf{c}_{3,3} \mathbf{y}_{3}$

subject to $\quad \mathbf{G}_{3} \mathbf{y}_{3} \leqq \mathbf{Y}_{3}+\mathbf{D}_{3} \mathbf{y}_{2}$,

$$
\mathbf{y}_{3} \geqq 0
$$

$<$ the reduced $(n-2)$ th level problem $>$

Maximize $\quad f_{n-1}\left(\mathbf{y}_{n-1}\right)=\mathbf{c}_{n-1} \mathbf{x}\left(\mathbf{y}_{n-1}\right)-\mathbf{c}_{n-1, n-1} \mathbf{y}_{n-1}$,

subject to $\quad \mathbf{G}_{n-1} \mathbf{y}_{n-1} \leqq \mathbf{Y}_{n-1}+\mathbf{D}_{n-1} \mathbf{y}_{n-2}$,

$$
\mathbf{y}_{n-1} \geqq 0
$$

$<$ the reduced $(n-1)$ th level problem $>$

Maximize $\quad f_{n}(\mathbf{x})=\mathbf{c}_{n} \mathbf{x}$,

subject to $\mathbf{A x}=\mathbf{b}+\mathbf{D}_{n} \mathbf{y}_{n-1}$

$\mathbf{x} \geqq 0$,

[Problem LP $\left.\mathbf{P}^{(n-1)}\right]$

Maximize $\quad f_{2}\left(\mathbf{y}_{2}, \mathbf{y}_{3}, \ldots, \mathbf{y}_{n-1}, \mathbf{x}\right)=\mathbf{c}_{2} \mathbf{x}-\mathbf{c}_{n-1,2} \mathbf{y}_{n-1}-\ldots-\mathbf{c}_{3,2} \mathbf{y}_{3}-\mathbf{c}_{2,2} \mathbf{y}_{2}$,

subject to $\quad \mathbf{G}_{i} \mathbf{y}_{i} \leqq \mathbf{Y}_{i}+\mathbf{D}_{i} \mathbf{y}_{i-1}, i=2,3, \ldots, n-1$,

$$
\begin{aligned}
& \mathbf{A x}=\mathbf{b}+\mathbf{D}_{n} \mathbf{y}_{n-1}, \\
& \mathbf{x}, \mathbf{y}_{2}, \mathbf{y}_{3}, \ldots, \mathbf{y}_{n-1} \geqq \mathbf{0},
\end{aligned}
$$

Problem $\mathbf{P}^{(n-1)}$ is constructed by combining the first and the second level problems in problem $\mathbf{P}^{(n)}$ excluding the objective function of the second level problem and $\mathbf{L} \mathbf{P}^{(n-1)}$ is constructed by combining the second level through the $n$th level problems excluding the objective functions at the third through the $n$th level problems.

Repeating this decomposition procedure, we can construct the $n-1$ multiobjectiveheadquarters, multi-level problems, $\mathbf{P}^{(n)}, \mathbf{P}^{(n-1)}, \ldots, \mathbf{P}^{(2)}$, and the $n-1$ single-level LP problems, $\mathbf{L} \mathbf{P}^{(n-1)}, \mathbf{L} \mathbf{P}^{(n-2)}, \ldots, \mathbf{L} \mathbf{P}^{(1)}$ and a multiobjective-headquarters, single-level problem $\mathbf{P}^{(1)}$. The lowest level problems $\mathbf{P}^{(1)}$ and $\mathbf{L} \mathbf{P}^{(1)}$ are finally derived as follows:

\section{[Problem $\left.\mathbf{P}^{(1)}\right]$}

Maximize $\quad f_{1}^{1}\left(\mathbf{y}_{1}, \mathbf{y}_{2}, \ldots, \mathbf{y}_{n-1}, \mathbf{x}\right)=\mathbf{c}_{1}^{1} \mathbf{x}-\mathbf{c}_{n-1,1}^{1} \mathbf{y}_{n-1}-\ldots-\mathbf{c}_{2,1}^{1} \mathbf{y}_{2}-\mathbf{c}_{1,1}^{1} \mathbf{y}_{1}$,

Maximize $\quad f_{1}^{L}\left(\mathbf{y}_{1}, \mathbf{y}_{2}, \ldots, \mathbf{y}_{n-1}, \mathbf{x}\right)=\mathbf{c}_{1}^{L} \mathbf{x}-\mathbf{c}_{n-1,1}^{L} \mathbf{y}_{n-1}-\ldots-\mathbf{c}_{2,1}^{L} \mathbf{y}_{2}-\mathbf{c}_{1,1}^{L} \mathbf{y}_{1}$,

subject to $\mathbf{G}_{1} \mathbf{y}_{1} \leqq \mathbf{Y}_{1}$,

$\mathbf{G}_{i} \mathbf{y}_{1} \leqq \mathbf{Y}_{i}+\mathbf{D}_{i} \mathbf{y}_{i-1}, i=2,3, \ldots, n-1$

$\mathbf{A x}=\mathbf{b}+\mathbf{D}_{n} \mathbf{y}_{n-1}$,

$\mathbf{x}, \mathbf{y}_{1}, \mathbf{y}_{2}, \ldots, \mathbf{y}_{n-1} \geqq \mathbf{0}$ 


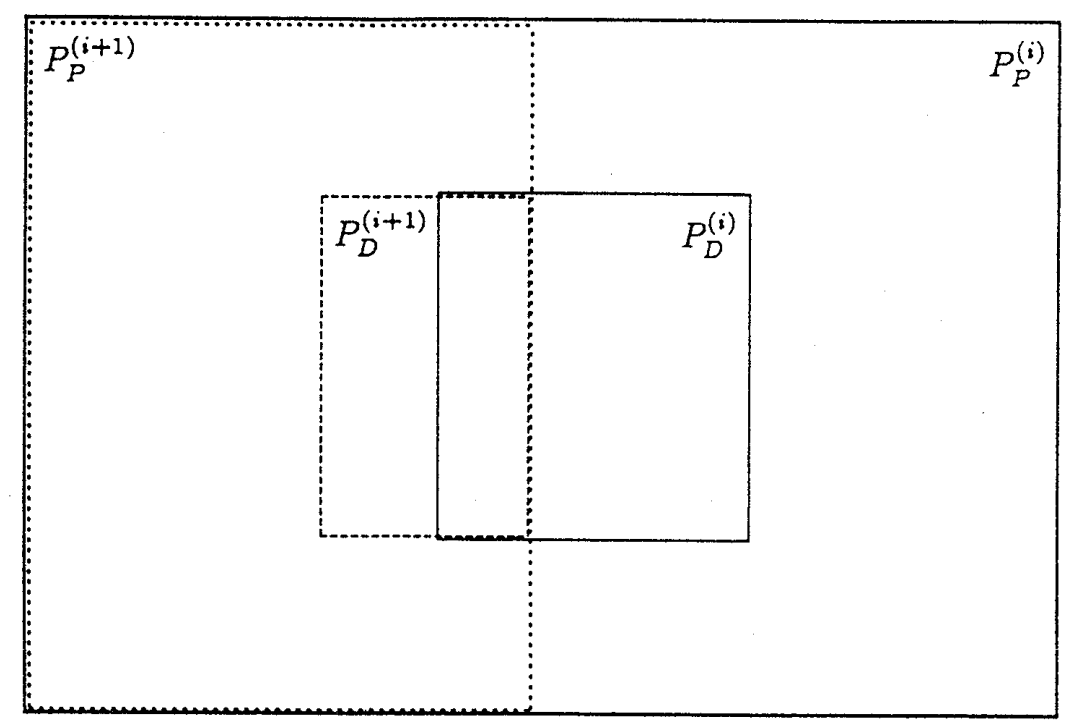

Fig. 2 Relationships among $P_{P}^{(i+1)}, P_{P}^{(i)}, P_{D}^{(i+1)}$ and $P_{D}^{(i)}$

[Problem LP(1)

Maximize $\quad f_{n}(\mathbf{x})=\mathbf{c}_{n} \mathbf{x}$

subject to $\mathbf{A x}=\mathbf{b}+\mathbf{D}_{n} \mathbf{y}_{n-1}$,

$\mathbf{x} \geqq 0$,

Let $P_{P}^{(i)}$ be the set of feasible extreme points in problem $\mathbf{P}^{(i)}$. Since any feasible solution can be expressed by a linear combination of some extreme points, any nondominated solution can be also represented by a linear combination of some feasible extreme points. Let $P_{D}^{(i)}$ be the set of nondominated extreme points to problem $\mathbf{P}^{(i)}$. Using linear programming theory and the results of analysis given by Wen [4] and Wang et al. [3], we can represent the relationships among these sets as follows:

$\left[\right.$ Property 1] $P_{D}^{(1)} \subseteq P_{P}^{(1)}$ and $P^{(i)} \subseteq P_{P}^{(i-1)}, i=2,3, \ldots n$.

[Property 2] If both $\mathbf{a} \in P_{P}^{(i+1)}$ and $\mathbf{a} \in P_{D}^{(i)}$ hold, then $\mathbf{a} \in P_{D}^{(i+1)}$ holds for $i=1,2, \ldots, n-1$. Conversely, if $\mathbf{a} \in P_{D}^{(i+1)}$ holds, then $\mathbf{a} \in P_{P}^{(i+1)}$ holds but $\mathbf{a} \in P_{D}^{(i)}$ does not always hold for $i=1,2, \ldots, n-1$.

[Property 3] If $\left(\mathbf{y}_{1}, \mathbf{y}_{2}, \ldots, \mathbf{y}_{n-1}, \mathbf{x}\right) \in P_{P}^{(i)}$ holds and if $\left(\mathbf{y}_{n-i+1}, \mathbf{y}_{n-i+2}, \ldots, \mathbf{y}_{n-1}, \mathbf{x}\right)$ optimizes problem $\mathbf{L} \mathbf{P}^{(i)}$ with resource $\mathbf{y}_{n-i}$, then $\left(\mathbf{y}_{1}, \mathbf{y}_{2}, \ldots, \mathbf{y}_{n-1}, \mathbf{x}\right) \in P_{P}^{(i+1)}$ for $i=$ $2,3, \ldots, n-1$.

For simplicity, consider a multiobjective, two level $(n=2)$ decentralized system. The original problem $\mathbf{P}^{(2)}$ defined by Eqs.(1) through (13) with $n=2$ is decomposed into problems $\mathbf{P}^{(1)}$ and $\mathbf{L} \mathbf{P}^{(1)}$ as defined by Eqs.(32) through (40) with $n=2$. It is obvious that $P_{D}^{(1)} \subseteq P_{P}^{(1)}$ and $P_{P}^{(2)} \subseteq P_{P}^{(1)}$. In problem $\mathbf{P}^{(2)}$, the second level problem gives the set of bases (the extreme points) with respect to $\mathbf{x}$ corresponding to the given vector $\mathbf{y}_{1}$ to the first level problem. The first level constructs the set of feasible extreme points by uniting the set of feasible extreme points with respect to $\mathbf{y}_{1}$ and the set of bases with respect to $\mathbf{x}$ 
provided from the second level problem. Nondominated extreme points are defined for this set of feasible extreme points and generated by searching each feasible extreme point in this set successively. Therefore, $P_{D}^{(2)} \subseteq P_{P}^{(2)}$ holds, that is, the nondominated extreme points are also feasible extreme points in problem $\mathbf{P}^{(2)}$.

According to properties 1 and 2 , the relationships among $P_{P}^{(i+1)}, P_{P}^{(i)}, P_{D}^{(i+1)}$ and $P_{D}^{(i)}$ can be illustrated in Fig. 2.

Using the "two-level simplex algorithm [3]", we can find all the elements of the set $P_{P}^{(2)}$. According to Property 3 , we can get all the elements of the set $P_{P}^{(n)}$ as the elements of $P_{P}^{(2)}$ satisfying the optimality conditions for problems $\mathbf{L} \mathbf{P}^{(1)}, \mathbf{L} \mathbf{P}^{(2)}, \ldots, \mathbf{L} \mathbf{P}^{(n-1)}$. Note that unlike the general multi-objective linear programming [5], it is impossible to judge whether or not each element in $P_{P}^{(2)}$ is nondominated for problem $\mathbf{P}^{(n)}$ by making a local judgement (i.e., solving a sub-LP problem) at each extreme point in $P_{P}^{(2)}$ on the searching process. This is because a nondominated solution to problem $\mathbf{P}^{(2)}$ is not always feasible for problem $\mathbf{P}^{(n)}$ and a dominated solution in problem $\mathbf{P}^{(2)}$ can be nondominated for problem $\mathbf{P}^{(n)}$ as indicated in Property 2. Therefore, if an element, $\mathbf{a}$, in $P_{D}^{(2)}$ does not belong to $P_{P}^{(n)}$, some extreme points dominated by the $\mathbf{a}$ in problem $\mathbf{P}^{(2)}$ can be nondominated solutions to problem $\mathbf{P}^{(n)}$. Fortunately, using Zeleny's theory of multi-objective linear programming [5], we can construct a set of domination trees which represent domination relationships between any pair of extreme points adjacent to each other in $P_{P}^{(2)}$. If an a in $P_{D}^{(2)}$ belongs to $P_{P}^{(n)}$, the $\mathbf{a}$ is nondominated solution to problem $\mathbf{P}^{(n)}$ according to Property 2 . Otherwise, all the extreme points dominated by the a in $P_{P}^{(2)}$ can be generated through the domination trees, and should be checked whether or not they belong to $P_{P}^{(n)}$. If we can find some extreme points belonging to $P_{P}^{(n)}$ on this checking process, then these points become candidates for nondominated solutions to problem $\mathbf{P}^{(n)}$. The detailed solution procedure is presented in section 4 .

In order to generate a set of domination trees, we have to derive additional properties with respect to extreme points in problem $\mathbf{P}^{(2)}$. For this purpose, we construct the following problem MLP which is equivalent to $\mathbf{P}^{(1)}$ :

\section{[Problem MLP]}

Minimize $\quad-f^{1}(\mathbf{z})=\mathbf{u}^{1} \mathbf{z}$,

Minimize $-f^{L}(\mathbf{z})=\mathbf{u}^{L} \mathbf{z}$,

subject to $\mathbf{Q z}=\mathbf{p}$,

$$
\mathbf{w} \geqq \mathbf{0}, \mathbf{y}_{i} \geqq \mathbf{0}, \mathbf{x}_{i j} \geqq \mathbf{0}, j=1,2, \ldots, n_{i}, i=1,2, \ldots, K,
$$

where $\mathbf{Q}, \mathbf{z}, \mathbf{p}$ and $\mathbf{u}^{l}$ (row vectors), $l=1,2, \ldots, L$, are defined by

$\mathbf{Q}=\left(\begin{array}{cccccccccccc}\mathbf{G}_{1} & \mathbf{I} & & & & & & & & & & \mathbf{O} \\ -\mathbf{D}_{2} & \mathbf{O} & \mathbf{G}_{2} & \mathbf{I} & & & & & & & & \\ & & -\mathbf{D}_{3} & \mathbf{O} & \mathbf{G}_{3} & \mathbf{I} & & & & & & \\ & & & & & & \ldots & & & & & \\ \mathbf{O} & & & & & & & -\mathbf{D}_{n-1} & \mathbf{O} & \mathbf{G}_{n-1} & \mathbf{I} & \\ -\mathbf{D}_{n} & \mathbf{O} & \mathbf{A}\end{array}\right)$

$\mathbf{z}=\left(\mathbf{y}_{1}^{T}, \mathbf{s}_{1}^{T}, \mathbf{y}_{2}^{T}, \mathbf{s}_{2}^{T}, \ldots, \mathbf{y}_{n-1}^{T}, \mathbf{s}_{n-1}^{T}, \mathbf{x}^{T}\right)^{T}$,

$\mathbf{p}=\left(\mathbf{Y}_{1}^{T}, \mathbf{Y}_{2}^{T}, \ldots, \mathbf{Y}_{n-1}^{T}, \mathbf{b}^{T}\right)^{T}$,

$\mathbf{u}^{l}=\left(\mathbf{c}_{1,1}^{l}, \mathbf{0}, \mathbf{c}_{2,1}^{l}, \mathbf{0}, \ldots, \mathbf{c}_{n-1,1}^{l}, \mathbf{0},-\mathbf{c}_{1}^{l}\right), l=1,2, \ldots, L$, 
where "T" denotes the transposition and $\mathbf{s}_{i}, i=1,2, \ldots, n-1$, are the slack variables for Eqs. (3), (6), (9).

Since the feasible region of problem MLP is made by combining Eqs. (3), (4), (6), (7), (9), (10), (12) and (13), any feasible solution in problem MLP satisfies condition (a). However, in problem MLP, the objective functions of the lower level problems are all removed and therefore a feasible solution in MLP does not always satisfy conditions (b) and (c).

A simplex tableau for problem MLP can be constructed by using the following equations:

$$
\begin{gathered}
\mathbf{z}_{B}+\left(\begin{array}{ccc}
\bar{q}_{1[m+1]} & \cdots & \bar{q}_{1[n]} \\
\vdots & & \vdots \\
\bar{q}_{m[m+1]} & \cdots & \bar{q}_{m[n]}
\end{array}\right) \mathbf{z}_{N}=\left(\begin{array}{c}
\bar{p}_{1} \\
\vdots \\
\bar{p}_{m}
\end{array}\right) \\
\left(\begin{array}{c}
\mathbf{u}^{1} \mathbf{z} \\
\vdots \\
\mathbf{u}^{L} \mathbf{z}
\end{array}\right)=\left(\begin{array}{c}
\mathbf{u}_{B}^{1} \\
\vdots \\
\mathbf{u}_{B}^{L}
\end{array}\right)\left(\begin{array}{c}
\bar{p}_{1} \\
\vdots \\
\bar{p}_{m}
\end{array}\right)+\left(\begin{array}{ccc}
\bar{u}_{[m+1]}^{1} & \cdots & \bar{u}_{[n]}^{1} \\
\vdots & & \vdots \\
\bar{u}_{[m+1]}^{L} & \cdots & \bar{u}_{[n]}^{L}
\end{array}\right) \mathbf{z}_{N}
\end{gathered}
$$

where $\bar{p}_{k}$ and $\bar{u}_{j}^{l}$ are defined as

$$
\begin{aligned}
& \bar{p}_{k}=\left(\mathbf{Q}_{B}^{-1} \mathbf{p}\right)_{k}, \bar{q}_{k[j]}=\left(\mathbf{Q}_{B}^{-1} \mathbf{Q}_{N}\right)_{k[j]}, \\
& \bar{u}_{[j]} \equiv\left(\mathbf{u}_{N}^{l}\right)_{[j]}-\left(\mathbf{u}_{B}^{l} \mathbf{Q}_{B}^{-1} \mathbf{Q}_{N}\right)_{[j]}, \overline{\mathbf{u}}_{j}=\left(\bar{u}_{j}^{1}, \bar{u}_{j}^{2}, \ldots, \bar{u}_{j}^{L}\right),
\end{aligned}
$$

where " $B$ " and " $N$ " stand for "basic" and "non-basic", respectively.

Letting $\Pi$ be an index set of nonbasic variables in the simplex tableau, we define

$$
\theta_{k}=\min _{1 \leq r \leq m}\left\{\frac{\bar{p}_{r}}{\bar{q}_{r k}} \mid \bar{q}_{r k}>0\right\}, k \in \Pi \text {. }
$$

Consider that the current extreme point in MLP belongs to $P_{P}^{(2)}$. Applying the results derived by Zenely [5] to this case, we get the following properties:

[Property 4] Assume $\theta_{j}>0$ and $\overline{\mathbf{u}}_{j} \leqq 0$ for a nonbasic variable, $z_{j}, j \in \Pi$. If the adjacent extreme point generated by introducing $z_{j}$ as a new basic variable belongs to $P_{P}^{(2)}$, then it dominates the current extreme point.

[Property 5] Assume $\theta_{j}>0$ and $\overline{\mathbf{u}}_{j} \geqq \mathbf{0}$ for a nonbasic variable, $z_{j}, j \in \Pi$. The adjacent extreme point generated by introducing $z_{j}$ as a new basic variable is dominated by the current extreme point.

[Property 6] Assume that $\theta_{j} \overline{\mathbf{u}}_{j} \geqq \theta_{k} \overline{\mathbf{u}}_{k}$ for two nonbasic variables $z_{j}$ and $z_{k}, j, k \in \Pi$. If the adjacent extreme point generated by introducing $z_{j}$ as a new basic variable belongs to $P_{P}^{(2)}$, then it dominates the adjacent extreme point generated by introducing $z_{k}$ as a new basic variable.

Using Properties 4 through 6 , we can construct a set of domination trees for $P_{P}^{(2)}$. Searching each extreme point downward from the top of each domination tree, we can find an extreme point ranked at the first highest level along a domination tree belonging to $P_{P}^{(n)}$. Then all the lower-ranked extreme points can be neglected without any search because they are explicitly dominated by the extreme point, reducing the computation time for checking whether they belong to $P_{P}^{(n)}$ or not. If we cannot find such an extreme point along a domination tree, all extreme points along the tree cannot become nondominated solutions to problem $\mathbf{P}^{(n)}$. 


\section{Solution Method}

We can not apply the existing methods developed for solving a usual multi-level system to generate a set of nondominated solutions to problem $\mathbf{P}^{(n)}$. We have to develop a new method exploiting Properties 1 through 6.

From Property 1 , any nondominated solution to problem $\mathbf{P}^{(n)}$, equivalently any element in $P_{D}^{(n)}$, not only belongs to $P_{P}^{(n)}$, but also belongs to $P_{P}^{(2)}$. However, from Property 2 , it does not always belong to $P_{D}^{(2)}$. Therefore, enumeration of all elements in $P_{D}^{(2)}$ itself does not solve problem $\mathbf{P}^{(n)}$. Additional information on the relationships among extreme points adjacent to each other in $P_{P}^{(2)}$, such as domination trees, is helpful to generate all the nondominated solutions to problem $\mathbf{P}^{(n)}$.

In the same way as that proposed for solving a single objective, two-level decentralized system, we can find all the extreme points in $P_{P}^{(2)}$ by using the "two-level simplex algorithm [3]." Incorporating Properties 4 through 6 into the two-level simplex algorithm, we can provide not only all the extreme points in $P_{P}^{(2)}$ but also the set of domination trees. If any nondominated solution to problem $\mathbf{P}^{(2)}$ listed at the top of each domination tree satisfies condition (b), then it belongs to $P_{D}^{(n)}$. Otherwise, the corresponding domination tree should be searched to find the first highest-ranked element along the domination tree satisfying condition (b). It should be noted that if the element satisfying condition (b) is not listed at the top of the corresponding domination tree, the element is not always a nondominated solution to problem $\mathbf{P}^{(n)}$, because it is not guaranteed for the element to satisfy condition (c). Condition (c) still remains to be checked at the last stage of the searching procedure. This final check can be achieved by comparing the values of headquarters-objective function vector of the element with those of all the elements generated.

We summarize the proposed algorithm for generating the set of nondominated solutions to problem $\mathbf{P}^{(n)}$ as follows:

$<$ Step $1>$ Make the set of domination trees over all the extreme points of $P_{P}^{(2)}$, by applying the two-level simplex algorithm to problem $\mathbf{P}^{(2)}$ and by using properties 4 through 6 . $<$ Step 2> Along each domination tree, find the first highest-ranked element satisfying the optimality conditions for problems $\mathbf{L} \mathbf{P}^{(2)}, \mathbf{L} \mathbf{P}^{(3)}, \cdots, \mathbf{L} \mathbf{P}^{(n-1)}$. If the domination tree has some branches, find such an element along each branch. If there is no such element found, abandon the tree or branch, and proceed the search for the other trees.

$<$ Step 3> Comparing the values of headquarters-objective function vector among the elements found in Step 2, generate the set of nondominated solutions to problem $\mathbf{P}^{(n)}$.

\section{Numerical Example}

To show the effectiveness and applicability of the proposed algorithm, we demonstrate a numerical example. Consider a corporation consisting of one headquarters and two division each of which has two factories. Assume that the corporation produces ten kinds of products. Each factory produces two or three kinds of product denoted by $\mathbf{x}_{11}=\left(x_{111}, x_{112}\right)^{T}$ for the $(1,1)$ factory, $\mathbf{x}_{12}=\left(x_{121}, x_{122}, x_{123}\right)^{T}$ for the $(1,2)$ factory, $\mathbf{x}_{21}=\left(x_{211}, x_{212}\right)^{T}$ for the $(2,1)$ factory and $\mathbf{x}_{22}=\left(x_{221}, x_{222}, x_{223}\right)^{T}$ for the $(2,2)$ factory. We can formulate the three-level decentralized system as follows:

[Example Problem $\mathbf{P}^{(3)}$ ]

$\leq$ the headquarters problem $>$

Maximize $\quad Z_{0}^{1}=(10,1) \mathbf{x}_{11}\left(\mathbf{y}_{1}(\mathbf{w})\right)+(4,1,1) \mathbf{x}_{12}(\mathbf{y}(\mathbf{w}))$ 


$$
\begin{gathered}
+(3,1) \mathbf{x}_{21}\left(\mathbf{y}_{2}(\mathbf{w})\right)+(1,4,4) \mathbf{x}_{22}\left(\mathbf{y}_{2}(\mathbf{w})\right) \\
-(8,5) \mathbf{y}_{1}(\mathbf{w})-(1,2) \mathbf{y}_{2}(\mathbf{w})-(1,1) \mathbf{w} \\
\text { Maximize } \quad \begin{array}{c}
2 \\
Z_{0}^{2}
\end{array}=(5,3) \mathbf{x}_{11}\left(\mathbf{y}_{1}(\mathbf{w})\right)+(4,1,1) \mathbf{x}_{12}\left(\mathbf{y}_{1}(\mathbf{w})\right) \\
+(2,3) \mathbf{x}_{21}\left(\mathbf{y}_{2}(\mathbf{w})\right)+(2,5,2) \mathbf{x}_{22}\left(\mathbf{y}_{2}(\mathbf{w})\right) \\
-(8,5) \mathbf{y}_{1}(\mathbf{w})-(1,2) \mathbf{y}_{2}(\mathbf{w})-(1,1) \mathbf{w}
\end{gathered}
$$

subject to $\left(\begin{array}{ll}2 & 2 \\ 4 & 3 \\ 3 & 1\end{array}\right) \mathbf{w} \leqq\left(\begin{array}{l}360 \\ 200 \\ 300\end{array}\right)$,

$$
\mathbf{w} \geqq \mathbf{0},
$$

$\leq$ the 1 st division problem $>$

Maximize $Z_{1}=(3,4) \mathbf{x}_{11}\left(\mathbf{y}_{1}\right)+(2,1,3) \mathbf{x}_{12}\left(\mathbf{y}_{1}\right)-(8,5) \mathbf{y}_{1}$,

subject to $\left(\begin{array}{cc}3 & 4 \\ 4 & 5 \\ 3 & 10\end{array}\right) \mathbf{y}_{1} \leqq\left(\begin{array}{c}36 \\ 18 \\ 30\end{array}\right)+\left(\begin{array}{cc}20 & 24 \\ 5.5 & 40 \\ 16 & 30\end{array}\right) \mathbf{w}$,

$$
\mathbf{y} \geq 0
$$

$\leq$ the $(1,1)$ factory problem $>$

Maximize $\quad Z_{11}=(2,3) \mathbf{x}_{11}$,

subject to $\left(\begin{array}{ll}2 & 0 \\ 3 & 3 \\ 1 & 3 \\ 3 & 4\end{array}\right) \mathbf{x}_{11} \leqq\left(\begin{array}{c}0 \\ 126 \\ 120 \\ 200\end{array}\right)+\left(\begin{array}{ll}2 & 0 \\ 0 & 2 \\ 0 & 0 \\ 0 & 0\end{array}\right) \mathbf{y}_{1}$,

$$
\mathbf{x}_{11} \geqq 0 \text {, }
$$

$\leq$ the $(1,2)$ factory problem $>$

Maximize $Z_{12}=(1,4,2) \mathbf{x}_{12}$,

subject to $\left(\begin{array}{ccc}4 & 4 & 2 \\ 8 & 10 & 10 \\ 10 & 20 & 5\end{array}\right) \mathbf{x}_{12} \leqq\left(\begin{array}{l}16 \\ 50 \\ 40\end{array}\right)+\left(\begin{array}{cc}0 & 0 \\ 0 & 0 \\ 0 & 1\end{array}\right) \mathbf{y}_{1}$,

$$
\mathbf{x}_{12} \geqq 0 \text {, }
$$

$\leq$ the 2nd division problem $>$

Maximize $Z_{2}=(1,2) \mathbf{x}_{21}\left(\mathbf{y}_{2}\right)+(5,9,3) \mathbf{x}_{22}\left(\mathbf{y}_{2}\right)-(1,2) \mathbf{y}_{2}$,

subject to $\left(\begin{array}{cc}9 & 4 \\ 6 & 5 \\ 3 & 10\end{array}\right) \mathbf{y}_{2} \leqq\left(\begin{array}{c}36 \\ 4 \\ 80\end{array}\right)+\left(\begin{array}{cc}28 & 20.5 \\ 40 & 18 \\ 20 & 10\end{array}\right) \mathbf{w}$,

$$
\mathbf{y}_{2} \geqq 0,
$$

$\leq$ the $(2,1)$ factory problem $>$

Maximize $Z_{21}=(1,3) \mathbf{x}_{21}$,

subject to $\left(\begin{array}{ll}2 & 0 \\ 3 & 3 \\ 1 & 3 \\ 3 & 2\end{array}\right) \mathbf{x}_{21} \leqq\left(\begin{array}{c}19 \\ 110 \\ 38 \\ 30\end{array}\right)+\left(\begin{array}{cc}0.5 & 0 \\ 0 & 1 \\ 0 & 2 \\ 0.5 & 0\end{array}\right) \mathbf{y}_{2}$,

$$
\mathbf{x}_{21} \geqq 0 \text {, }
$$

$\leq$ the $(2,2)$ factory problem $>$

Maximize $Z_{22}=(1,1,1) \mathbf{x}_{22}$,

subject to $\left(\begin{array}{lll}2 & 4 & 2 \\ 2 & 1 & 1 \\ 2 & 2 & 5\end{array}\right) \mathbf{x}_{22} \leqq\left(\begin{array}{c}60 \\ 80 \\ 5\end{array}\right)+\left(\begin{array}{cc}0.5 & 0 \\ 1 & 0 \\ 0.5 & 1\end{array}\right) \mathbf{y}_{2}$,

$$
\mathbf{x}_{22} \geqq 0
$$

Using the algorithm proposed in section 4 , we can find all the extreme points in $P_{P}^{(2)}$ with the domination trees as shown in Table 1 and Fig. 3. After checking whether or not 


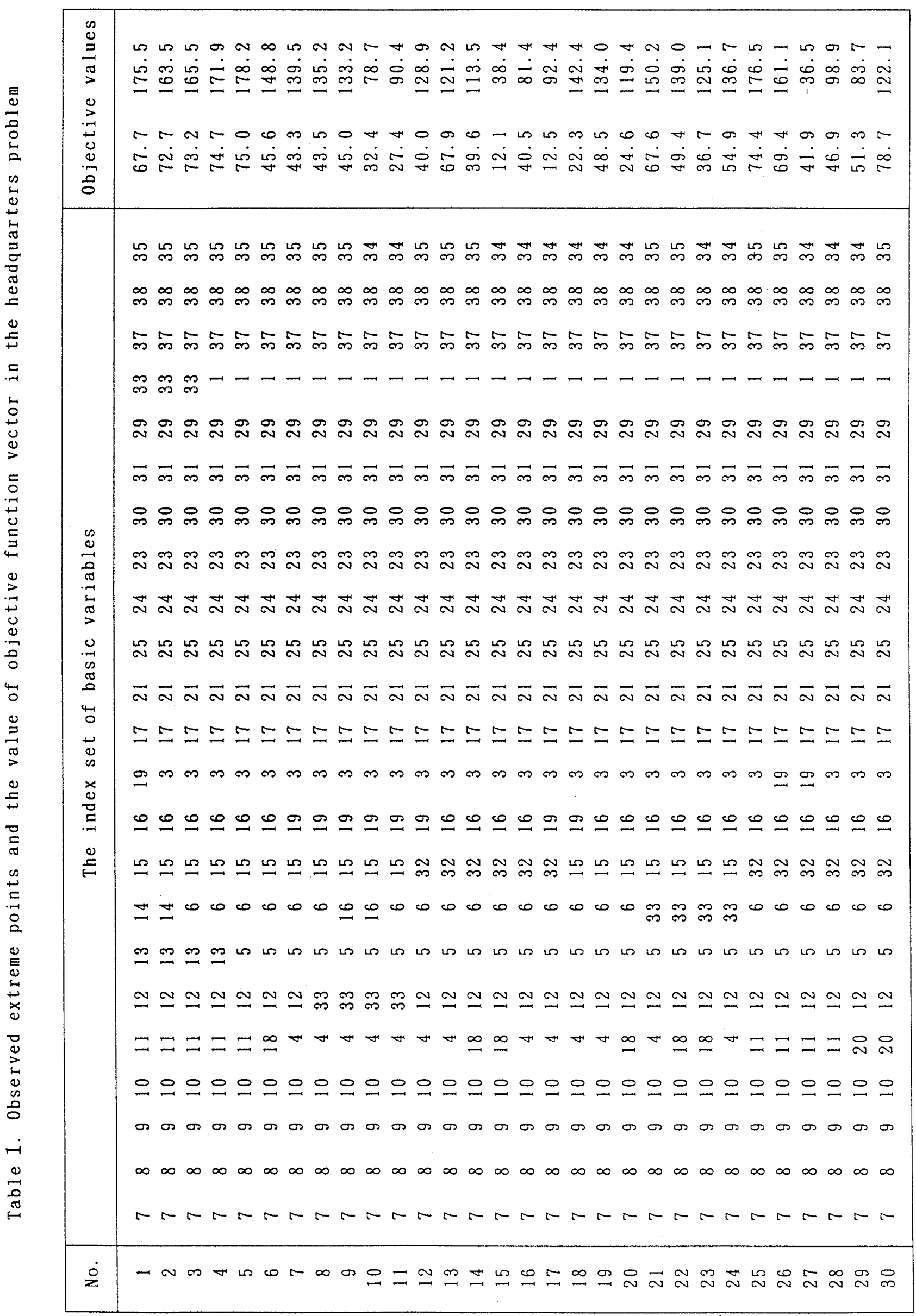




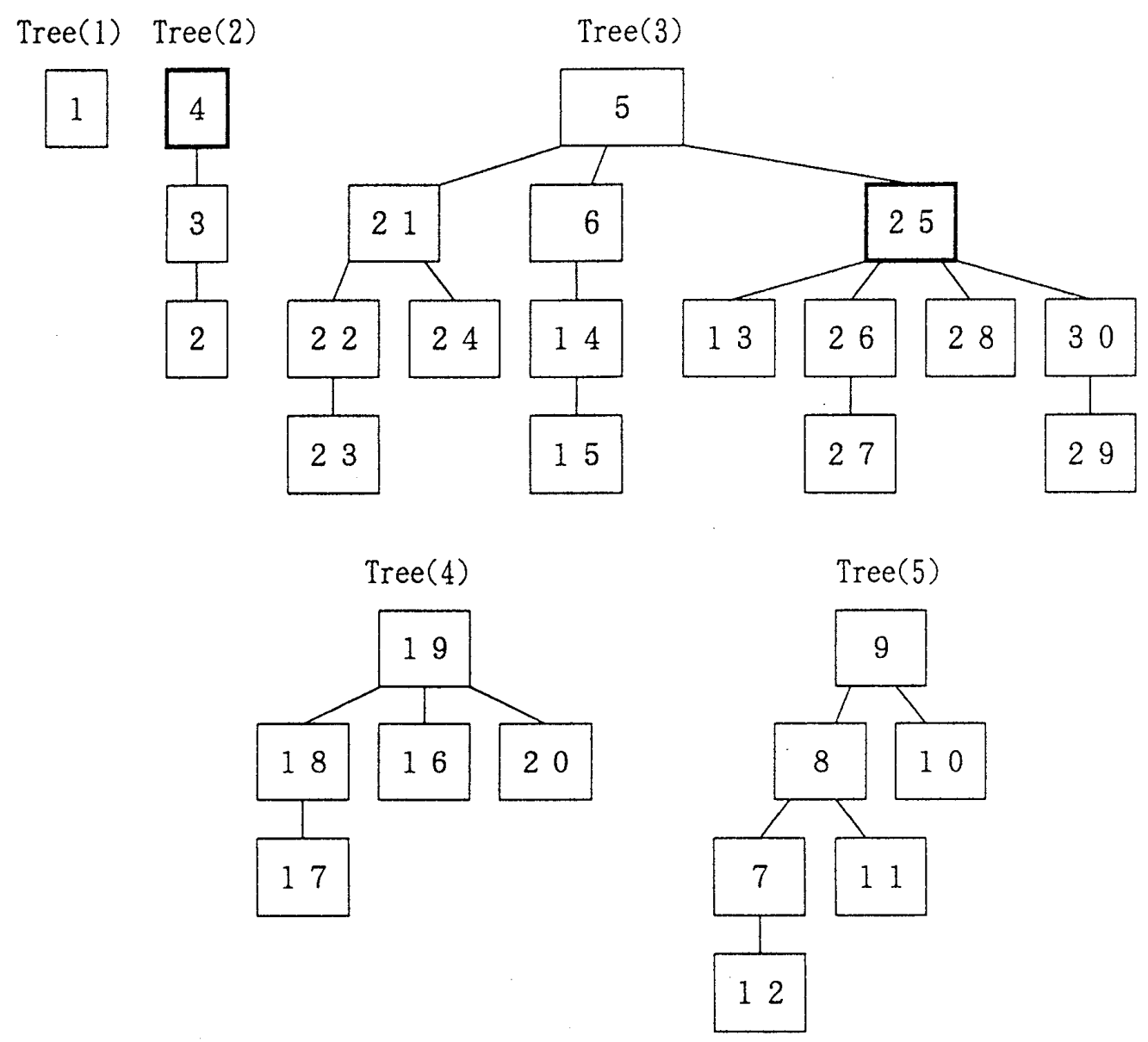

Fig. 3 Domination trees for the example problem

these elements satisfy the condition (b), we finally obtained elements 4 and 25 along trees (2) and (3). The other trees have no element satisfying condition (b). Comparing the values of headquarters-objective-function vector among these two elements, we get elements 4 and 25 as the set of nondominated solutions to problem $\mathbf{P}^{(3)}$. In this case, 30 elements in $P_{P}^{(2)}$ are generated, but only 22 elements are checked to judge whether or not satisfy condition (b). Domination trees are helpful to reduce a large amount of computation effort for achieving this final check. Computation time is only about 1 minute by EPSON PC-286 without a mathematical processor.

\section{Conclusions}

This paper discussed a mathematical model and its solution method for a multi-objective, multi-level decentralized system. An optimal solution method based on the two-level simplex algorithm with domination trees was proposed for solving this type of multi-level decentralized system. A numerical example was demonstrated to show the effectiveness of the proposed method.

\section{References}

[1] Anandalingam, G: A mathematical Programming Model of Decentralized Multi-Level systems. J. Operational Research Society, 39(1988), 1021-1033. 
[2] Wang, Z. W., H. Nagasawa and N. Nishiyama, Optimal Resource Allocation in a Threelevel Decentralized System. Bulletin of University of Osaka Prefecture, Series A, 41(1992), no. $5,57-68$.

[3] Wang, Z. W., H. Nagasawa and N. Nishiyama, A New Method for Solving a Linear Two-level Decentralized Problem. J. of the Operations Research Society of Japan, Vol. 38, No. 3(1995), 345-354, in Japanese.

[4] Wen, U. P. and S. T. Hsu, Linear Bi-level Programming Problems - A Review. J. of the Operational Research Society, Vol. 42, No. 2(1991), 125-133.

[5] Zeleny, M., Linear Multiobjective Programming. Chapter 3, Springer-Verlag, Berlin and N.Y., 1974.

Hiroyuki Nagasawa

Department of Industrial Engineering

College of Engineering

Osaka Prefecture University

1-1 Gakuen-cho, Sakai, Osaka, 580, Japan

E-mail: ng@ie.osakafu-u.ac.jp 